\title{
Author Index Vol. 63, 1994
}

Alexander, J.P. 59 Anapol, F. 177 Bibollet-Ruche, F. 226 Bicca-Marques, J.C. 209,216 Bonnaire, L. 171 Brumloop, A. 212 Calegaro-Marques, C. 209,216 Chapman, C.A. 181 Cordeiro, N.J. 84 D’Ovidio,M.C. 221 Duckworth, J.W. 99 Durand,J.P. 226 Ferrari, S.F. 78 Galat,G. 226 Galat-Luong, A. 226 Ganzhorn, J.U. 203 Homburg, I. 212 Jolly, CJ. 177 Judge, P.G. 63 Julliot,C. 75 Kaplan, G. 50 Kappeler, P.M. 162 Kirkpatrick, R.C. 102 Kleiman,D.G. 131 Krishnamurthy, R. 91 Laska, M. 144 Long, Y.C. 102 Macedonia, J.M. 1 McLean, S.P. 107 Maestripieri, D. 192 Marzke,M. 107 Masataka, N. 115

Pace,M. 221 Peetz,A. 212 Pichon,G. 226 Pourrut,X. 226 Power, M.L. 131 Rakotoarison, N. 156 Riehl,R. 212 Rogers, L.J. 50 Ron, T. 71 Rutledge, C.E. 131 Rylands, A,B. 78 Santini-Palka, M.E. 44 Sarni,P. 226 Schmid, J. 162 Schmitt,J. 123 Simmen, B. 171 Stanger,K,F. 1 Strom, R. 221 Suomi,SJ. 96 Tardieu, C. 53 Thalmann, U. 156 Thompson, S.D. 131 Tomiuk, J. 123 Turillazzi, P.G. 221 Turner, T.R. 177 Via,F. 221 Vitale,A. 88 Waal, F.B.M. de 63 Westergaard, G.C. 96 White, F.J. 181 Wright, P.C. 203 